\title{
Conhecimentos sobre prevenção e tratamento de glaucoma entre pacientes de unidade hospitalar
}

\author{
Knowledge on glaucoma prevention and treatment of patients in a hospital unit
}

\author{
Marcelo Jordão Lopes da Silva1 \\ Edméa Rita Temporini ${ }^{2}$ \\ Isaac Neustein ${ }^{3}$ \\ Maria Emília Xavier Santos Araújo ${ }^{4}$
}

Trabalho realizado no Hospital do Servidor Público Estadual - SP.

${ }^{1}$ Médico Oftalmologista, Mestre em Medicina pelo IASMPE, Chefe do Setor de Glaucoma do Hospital do Servidor Público Estadual, São Paulo.

2 Professora Associada, Livre-docente em Metodologia de Pesquisa em Saúde, Assessora de Pesquisa junto à Disciplina de Oftalmologia da Faculdade de Medicina da Universidade de São Paulo - USP e da Faculdade de Ciências Médicas da Universidade Estadual de Campinas - UNICAMP.

${ }^{3}$ Médico Oftalmologista, Chefe do Serviço de Oftalmologia do Hospital do Servidor Público Estadual, São Paulo.

${ }^{4}$ Médica Oftalmologista, Doutora em Oftalmologia pela Universidade Federal de São Paulo - UNIFESP.

Endereço para correspondência: Marcelo Jordão Lopes da Silva, Rua Martinico Prado, 417 apto 62 - São Paulo (SP) CEP 01224-010

E-mail: marcelojordao@webcable.com.br

Nota Editorial: Pela análise deste trabalho e por sua anuência na divulgação desta nota, agradecemos ao Dr. Maurício Della Paolera.

Recebido para publicação em 04.07.2003

Versão revisada recebida em 26.03.2004

Aprovação em 22.04.2004

\begin{tabular}{|l|}
\hline RESUMO \\
\hline Objetivo: Verificar conhecimentos de pacientes portadores de glaucoma \\
em relação a sua afecção, com a finalidade de obter subsídios para auxiliar \\
a relação médico-paciente e estimular a observância do tratamento. Méto- \\
dos: No Hospital do Servidor Público Estadual do município de São Paulo, \\
Brasil, foi realizado estudo transversal analítico aplicando-se questionário \\
estruturado, com base em estudo exploratório e submetido a teste prévio \\
para avaliação do nível conhecimento em relação ao glaucoma. A variável \\
"auto-avaliação do conhecimento" foi mensurada por escala ordinal (sabe \\
bem, sabe mais ou menos, sabe mal e nada sabe). Resultados: A população \\
foi constituída por 405 pacientes portadores de glaucoma; 72,6\% do sexo \\
feminino; idade média 66,2 anos; $54,3 \%$ cursaram até o ensino fundamental. \\
Os resultados revelaram: dos que sabem bem sobre o controle da doença, \\
95, $8 \%$ declararam terem recebido explicações (p < 0,000); houve maior \\
proporção (46,9\%) de pacientes que afirmaram "saber mais ou menos" \\
quando comparado com os demais grupos, porém aqueles com maior \\
escolaridade referiram maior conhecimento quando comparado aos com \\
menor escolaridade (p <0,000); em relação às fontes de informação sobre \\
glaucoma49,9\% mencionaram unicamenteo oftalmologista. Conclusão: O \\
conhecimento dos pacientes em relação ao glaucoma foi relacionado às \\
explicações recebidas e ao nível de escolaridade. Este estudo confirma a \\
necessidade da manutenção de orientações, divulgação continuada de \\
informações sobre prevenção e tratamento de glaucoma, nos consultórios \\
e na comunidade, para melhora do prognóstico visual. \\
\hline
\end{tabular}

Descritores: Glaucoma/terapia; Glaucoma/prevenção \& controle; Educação do paciente; Saúde pública/educação; Cegueira/prevenção \& controle; Hospitais públicos

\section{INTRODUÇÃOO}

O glaucoma é uma afecção que, por suas características clínicas e prognóstico visual, requer comprometimento do paciente com o tratamento. Trata-se de doença crônica que deve receber acompanhamento e tratamento prolongado, condições estas que previnem a cegueira.

Os oftalmologistas apresentam papel importante na detecção precoce do glaucoma e na sua desmistificação ${ }^{(1)}$. O desconhecimento sobre a doença e a elaboração de idéias falsas tendem a gerar ausência de participação do paciente no tratamento, agravando o prognóstico visual ${ }^{(2)}$.

$\mathrm{O}$ conceito de glaucoma tem mudado ao longo dos anos podendo ser definido atualmente como neuropatia óptica crônica, caracterizada por perda de campo visual e lesão do nervo óptico ${ }^{(3)}$. Portanto, a pressão intra-ocular já não é mais tida como fator indispensável para a ocorrência da afecção. 
O aumento da PIO é considerado como um dos fatores de risco, portanto, na etiopatogenia do glaucoma outros fatores somam-se a ela, acarretando pior prognóstico da doença; entre eles, o desconhecimento da população a respeito da doença e suas conseqüências visuais ${ }^{(4)}$. Assim, estudos referiram altos índices de cegueira unilateral $(51,8 \%)$ e bilateral $(33,3 \%)$ de glaucomatosos atendidos pela primeira vez no Setor de Glaucoma da Universidade de Campinas ${ }^{(5)}$.

A falta de observância do tratamento pelo paciente foi identificada como causa importante de controle inadequado de pressão intra-ocular ${ }^{(6)}$ (PIO), tendo-se ressaltado, que até $50 \%$ dos pacientes deixaram de tomar medicamentos antiglaucomatosos corretamente ${ }^{(7)}$.

Analisando o conhecimento de glaucomatosos sobre a doença e o tratamento, estudos observaram que a população investigada estava desinformada sobre suas condições clínicas, a doença, tratamento e métodos para o diagnóstico e monitorização do glaucoma ${ }^{(8)}$.

O conhecimento em relação à doença ocular constitui condição necessária e antecede as ações do indivíduo para preservar a visão. Embora se reconheça que a ampliação e melhoria do conhecimento per se nem sempre resulte em mudança comportamental, as atitudes, crenças, valores e percepções do indivíduo, compõe o grupo de fatores responsáveis pela justificativa ou motivação para recusar ou adotar um comportamento específico. Esses fatores incluem dimensões cognitivas e afetivas do saber, sentir, acreditar, valorizar e da autoconfiança ou percepção da própria competência para agir de determinada maneira ${ }^{(9)}$.

Tais aspectos devem merecer especial atenção na medida em que se pretenda obter um diagnóstico prévio da realidade, antecedendo alguma intervenção. Desse modo, pode-se direcionar apropriadamente o planejamento de ações de saúde, tendo em vista grupos-alvo diversos ${ }^{(10)}$.

Ações educativas na relação médico-paciente constroem a base para a promoção da saúde ocular e a preservação do sistema visual, aumentando a capacidade dos indivíduos de tomar decisões relativas a comportamentos que influenciarão seu nível de saúde ocular ${ }^{(11)}$.

A experiência tem mostrado que nem sempre o fácil acesso a serviços educativo-assistenciais assegura, por si só, um nível de saúde satisfatório de uma população. Influências restritivas interferem na tomada de decisões e na execução de ações de saúde, decorrentes de fatores psicossocioculturais. A importância que as pessoas conferem a sua saúde e aos cuidados para preservá-la depende, em especial, de padrões socioeconômicos, de conhecimentos, hábitos, atitudes e crenças aprendidos culturalmente ${ }^{(12)}$.

A importância de se investigar esse tema reside no fato de que a população brasileira, em sua maioria, tem acesso insuficiente a informações adequadas em relação à saúde em geral e sobre doenças crônicas como o glaucoma. Supõe-se que esse desconhecimento acarreta baixa adesão ao tratamento e conseqüente comprometimento do prognóstico visual.
Desse modo, o objetivo deste estudo consistiu em verificar informações recebidas em relação ao glaucoma e o grau de auto-avaliação de conhecimentos, referentes a essa afecção, na percepção de pacientes portadores de glaucoma, atendidos em unidade hospitalar. Considerou-se assim, a possibilidade de obter subsídios que possam facilitar a relação médicopaciente e estimular a observância do tratamento proposto.

\section{MÉTODOS}

Realizou-se pesquisa de corte transversal entre 405 indivíduos portadores de glaucoma, atendidos no ambulatório do Setor de Glaucoma do Hospital do Servidor Público Estadual de São Paulo (HSPE), no município de São Paulo. A amostra foi obtida a partir dos seguintes critérios: idade de 18 anos ou mais, presença a retorno no ambulatório no período estabelecido para a coleta de dados e concordância em participar deste estudo.

Foi elaborado questionário com base em estudo preliminar da realidade - denominado estudo exploratório. Esse recurso metodológico apresenta a finalidade de obter informações a respeito de terminologia, expressões verbais e variáveis, presentes em população similar. O conhecimento assim obtido permite introduzir no questionário da pesquisa elementos que integram aquela realidade, o que facilita a comunicação com os sujeitos da amostra e a compreensão das questões do instrumento. O estudo exploratório leva o pesquisador, freqüentemente, a descobertas de enfoques, percepções e terminologias novas para ele, contribuindo para que, paulatinamente, seu próprio modo de pensar seja modificado ${ }^{(13)}$.

Este estudo foi realizado em duas fases, nas quais as informações foram obtidas de forma gradual, por meio de entrevistas e aplicação de roteiro de perguntas.

Da primeira fase participaram 100 pacientes que forneceram elementos esclarecedores a respeito da relevância dos aspectos indagados, dúvidas quanto às perguntas formuladas e termos difíceis de serem compreendidos. Na segunda fase, foi aplicado questionário semi-estruturado a 20 pacientes, construído a partir dos subsídios obtidos na fase anterior. Após análise dessas entrevistas, elaborou-se o questionário definitivo.

O questionário assim construído foi submetido a teste prévio, mediante a aplicação por entrevista a 120 sujeitos, como prova de validade e confiança. Os pacientes que participaram do teste prévio foram excluídos da amostra do estudo.

O presente estudo apresenta as seguintes variáveis: sexo, idade, escolaridade, recebimento de explicações sobre glaucoma e auto-avaliação de conhecimentos em relação ao glaucoma. A variável "auto-avaliação do conhecimento" foi mensurada por meio de escala ordinal, incluindo as categorias: sabe bem, sabe mais ou menos, sabe mal e nada sabe. Desse modo procurou-se aumentar a precisão da medida. A adequação dessa escala confirmou-se nas fases exploratória e do teste prévio do instrumento. 
A coleta de dados foi realizada no período de dezembro de 2001 a maio de 2002, mediante a aplicação do questionário por entrevista, considerando possíveis limitações da população de estudo decorrentes de baixa escolaridade. As entrevistas foram realizadas por auxiliar de pesquisa previamente treinada. Foram assegurados anonimato e sigilo dos dados aos respondentes.

A análise estatística foi realizada por meio do teste quiquadrado, estabelecido o nível de significância de 0,05.

\section{RESULTADOS}

A amostra foi composta por 405 pacientes de idades entre 20 e 92 anos.

$\mathrm{Na}$ tabela 1 apresentam-se as características pessoais. Observa-se que a maioria era do sexo feminino $(72,6 \%)$ e que a idade média foi de 66,17 anos e desvio padrão $\pm 10,85$ anos. Quanto à escolaridade, 54,3\% cursaram até o ensino fundamental.

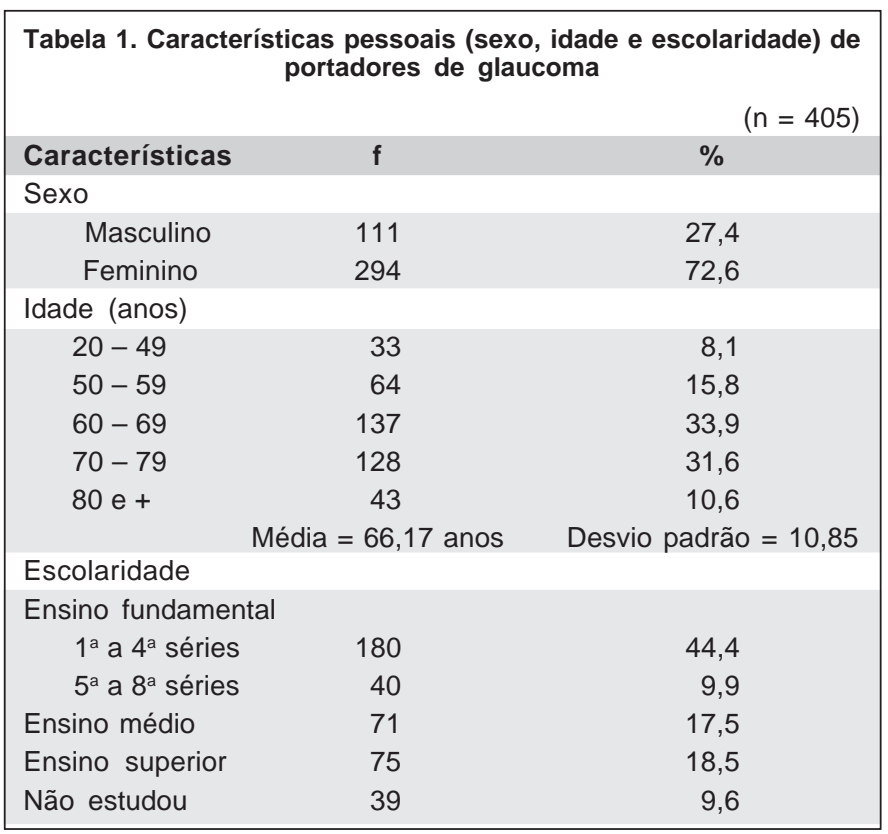

A tabela 2 mostra a relação entre a escolaridade e a autoavaliação do conhecimento sobre glaucoma. Os resultados mostraram que uma maior porcentagem dos pacientes entrevistados $(46,9 \%)$ acreditavam "saber mais ou menos", entretanto, observou-se que entre aqueles com maior escolaridade referiram conhecer mais do que os com menor escolaridade $(\mathrm{p}<0,000)$.

A tabela 3 apresenta o cruzamento das respostas dadas pelos pacientes quando indagados sobre as explicações recebidas e a auto-avaliação do conhecimento sobre glaucoma. Nota-se que há maior proporção de entrevistados que responderam afirmativamente em relação ao recebimento de explicações, entre aqueles que declaram saber bem o que é glaucoma do que entre os demais grupos $(\mathrm{p}<0,05 \%)$. Em relação ao conhecimento do controle do glaucoma, entre os que declaram conhecer bem a doença, 95, 8\% declaram terem recebido explicações $(\mathrm{p}<0,000)$; entre os que declaram ter recebido tais explicações também afirmam conhecer a finalidade do colírio, o conceito e razão do exame de campo visual $(\mathrm{p}<0,000)$.

Em relação às fontes de informação a respeito de glaucoma, 49,9\% dos entrevistados mencionam unicamente o médico oftalmologista. De forma associada, mencionam médico e material impresso, sob a forma de jornais, revistas e livros $(7,2 \%)$ (Tabela 4).

\section{DISCUSSÃO}

O glaucoma é a segunda maior causa de cegueira no mundo. Estimou-se que no ano de 2000, 67 milhões de pessoas seriam portadoras de glaucoma primário de ângulo aberto ou fechado e que $10 \%$ destas seriam bilateralmente cegas ${ }^{(14)}$.

Sabe-se que aproximadamente metade dos casos de cegueira é previsível ou reversível, tanto nos $\mathrm{EUA}^{(15)}$ como no Brasil $^{(16)}$. Estudos apontaram que um ano de cegueira em um indivíduo adulto produtivo custava ao governo norte-americano cerca de U\$12.000, em 1990 ${ }^{(17)}$. Além disso, o custo da prevenção da cegueira é muito menor que o custo social causado pela cegueira ${ }^{(18)}$.

No presente estudo, investigaram-se informações recebidas em relação ao glaucoma e o grau de auto-avaliação de conhecimentos, na percepção de pacientes portadores dessa

\begin{tabular}{|c|c|c|c|c|c|}
\hline \multirow[b]{3}{*}{ Escolaridade } & & & & & \multirow[t]{2}{*}{$(n=405)$} \\
\hline & \multicolumn{4}{|c|}{ Auto-avaliação do conhecimento sobre glaucoma } & \\
\hline & $\begin{array}{c}\text { Sabe bem } \\
(n=24) \\
\%\end{array}$ & $\begin{array}{c}\text { Sabe mais ou menos } \\
(n=190) \\
\%\end{array}$ & $\begin{array}{c}\text { Sabe mal } \\
(n=116) \\
\%\end{array}$ & $\begin{array}{c}\text { Nada sabe } \\
(\mathrm{n}=75) \\
\%\end{array}$ & Valor de $p$ \\
\hline De $1^{a}$ a $4^{a}$ série & 2,8 & 46,7 & 26,1 & 24,4 & 0,000 \\
\hline De $5^{\mathrm{a}}$ a $8^{\mathrm{a}}$ série & 10,0 & 50,0 & 30,0 & 10,0 & \\
\hline Ensino médio & 4,2 & 52,1 & 29,6 & 14,1 & \\
\hline Ensino superior & 16,0 & 46,7 & 32,0 & 5,3 & \\
\hline Não estudou & - & 35,9 & 30,8 & 33,3 & \\
\hline
\end{tabular}


afecção, com a finalidade de obter subsídios para auxiliar a relação médico-paciente e estimular a observância do tratamento.

Observou-se, um maior valor percentual de pacientes que afirmaram "saber mais ou menos" em relação ao glaucoma, porém, entre aqueles com maior escolaridade referiram "saber mais", ou seja, pacientes com menor escolaridade confirmam seu desconhecimento a respeito de sua afecção.

Estudos observaram que pacientes mais bem informados apresentaram melhor comprometimento com o tratamento ${ }^{(19)}$, e que em tratamento prolongado, sem compreensão da sua finalidade e importância pelos pacientes, tornava-se difícil a participação no tratamento $^{(2)}$. Os glaucomatosos ao serem questionados sobre a freqüência com que usavam os medicamentos, tendiam a repetir os intervalos dos horários indicados pelo médico. Entretanto, ao serem interrogados sobre os horários, percebeu-se que esses intervalos não eram respeitados. Apesar disso, muitos confiavam que, com o uso dos colírios, poderia haver melhora da acuidade visual. Como tal fato não ocorre, também contribui para reduzir a adesão ao tratamento.

Outros fatores podem estar também relacionados à irregularidade do tratamento, como: dificuldades econômicas, esquecimento do horário dos medicamentos, ausência de melhora da visão, efeitos colaterais e dificuldade da auto-instilação dos colírios ${ }^{(2)}$.

Ley observou que os pacientes tendem a esquecer de um terço à metade das informações, minutos após o recebimento ${ }^{(20)}$. Outros autores investigaram o efeito de um vídeo de 6 minutos

Tabela 3. Auto-avaliação do conhecimento sobre glaucoma segundo o recebimento de explicações sobre glaucoma entre portadores de glaucoma

\begin{tabular}{|c|c|c|c|c|c|c|}
\hline \multirow[b]{2}{*}{ Recebimento de explicações } & \multicolumn{6}{|c|}{ Auto-avaliação do conhecimento sobre glaucoma } \\
\hline & & $\begin{array}{c}\text { Sabe bem } \\
(n=24) \\
\%\end{array}$ & $\begin{array}{l}\text { Sabe mais ou menos } \\
\qquad \begin{array}{c}(n=190) \\
\%\end{array}\end{array}$ & $\begin{array}{c}\text { Sabe mal } \\
(n=116) \\
\%\end{array}$ & $\begin{array}{c}\text { Nada sabe } \\
(n=75) \\
\%\end{array}$ & Valor de $p$ \\
\hline \multirow[t]{2}{*}{ Controle do glaucoma } & Não & 4,2 & 24,7 & 31,0 & 60,0 & 0,000 \\
\hline & Sim & 95,8 & 75,3 & 69,0 & 40,0 & \\
\hline Uso do colírio & Não & 25,0 & 29,5 & 33,6 & 50,7 & 0,008 \\
\hline \multirow[t]{2}{*}{ Finalidade do uso do colírio } & Não & 29,2 & 34,7 & 44,0 & 66,7 & 0,000 \\
\hline & $\operatorname{Sim}$ & 70,8 & 65,3 & 56,0 & 33,3 & \\
\hline \multirow{2}{*}{$\begin{array}{l}\text { Necessidade exame oftalmológico } \\
\text { na família }\end{array}$} & Não & 41,7 & 58,9 & 67,2 & 76,0 & 0,006 \\
\hline & $\operatorname{Sim}$ & 58,3 & 41,1 & 32,8 & 24,0 & \\
\hline \multirow[t]{2}{*}{ Razão da cirurgia do glaucoma } & Não & 54,2 & 63,7 & 71,6 & 81,3 & 0,014 \\
\hline & Sim & 45,8 & 36,3 & 28,4 & 18,7 & \\
\hline Conceito do exame de campo visual & $\operatorname{Sim}$ & 50,0 & 22,1 & 8,6 & 6,7 & \\
\hline \multirow[t]{2}{*}{ Razão do exame de campo visual } & Não & 45,8 & 73,2 & 88,8 & 90,7 & 0,000 \\
\hline & Sim & 54,2 & 26,8 & 11,2 & 9,3 & \\
\hline
\end{tabular}

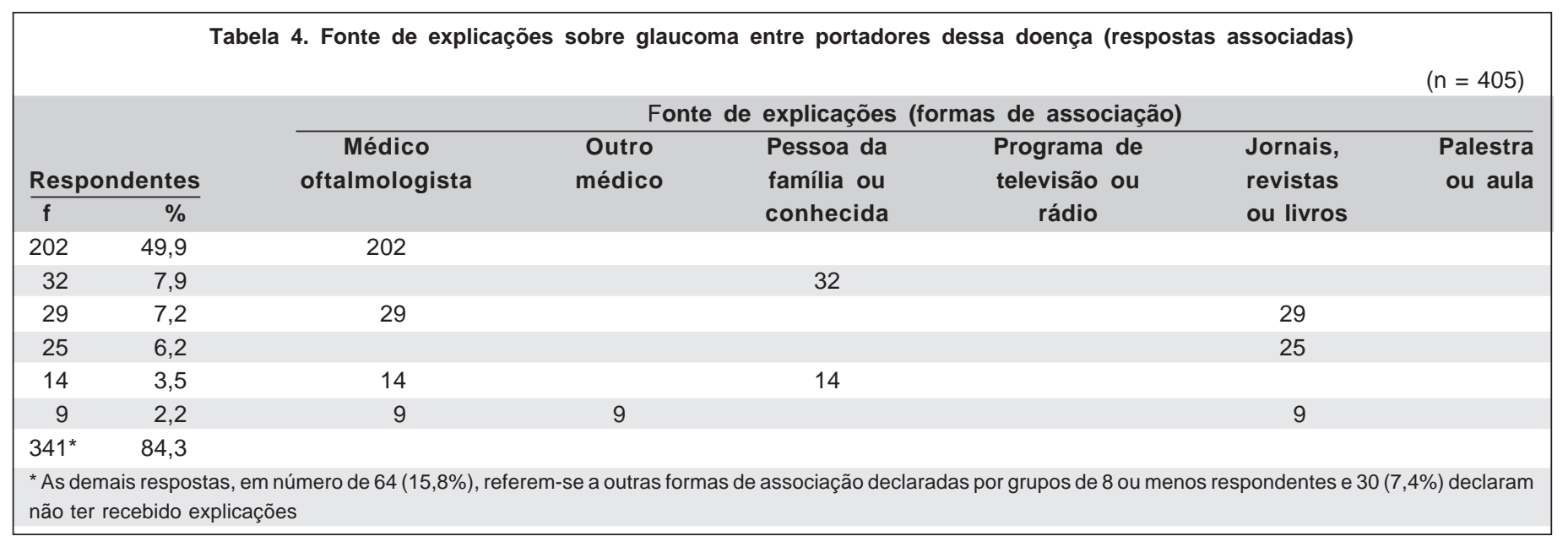


sobre o conhecimento a respeito do glaucoma. Apesar de ter sido observado melhor conhecimento imediatamente após a apresentação do vídeo, após seis meses houve queda importante do nível de conhecimento ${ }^{(21)}$. Kim et al. demonstraram que um vídeo de 12 minutos produzido pela Academia Americana de Oftalmologia promoveu melhora do conhecimento sobre glaucoma após uma semana, mas não após três meses ${ }^{(22)}$.

Esses fatos reforçam a necessidade da manutenção de orientações e divulgação continuada de informação sobre prevenção e tratamento de glaucoma nos consultórios e na comunidade.

A aquisição do conhecimento sobre a importância da hereditariedade é muito importante para alertar descendentes sobre o risco de desenvolver o glaucoma.

A relação médico-paciente mostrou-se a mais expressiva fonte de informações, associado a jornais, revistas ou livros. É interessante observar que campanhas em televisão, rádio, meios de comunicação em massa, não se mostraram importantes na transmissão de conhecimentos para os entrevistados.

Admite-se que para realização de ações de saúde pública que dependem do comportamento das pessoas a que se destinam, torna-se de extrema importância conhecer previamente as maneiras de agir, sentir e pensar da comunidade-alvo dessas ações e o contexto onde se insere essa comunidade ${ }^{(23)}$. Esse conhecimento possibilita direcionar o conteúdo e forma de campanhas educativas visando à prevenção e controle do glaucoma com objetivo, no caso, de maior compreensão e aceitação dos pacientes glaucomatosos da importância da adesão ao tratamento e controle adequado da doença.

Partindo do pressuposto que o conhecimento não corresponde a um acúmulo gradual de informações transmitidas, mas sim, a um processo construído na interação entre o sujeito e o outro, Cintra et al. ${ }^{(2)}$ sugeriram que a orientação dos portadores de glaucoma deve ser feita de forma contínua e progressiva, levando-se em conta as concepções e significados que atribuem a sua doença e tratamento.

Considera-se, portanto, ser tarefa da equipe de saúde a identificação do repertório de conhecimentos e vocábulos comumente empregados pelas pessoas da comunidade. Este constitui o primeiro passo para uma comunicação eficiente ${ }^{(13)}$.

É sempre importante enfatizar que o modelo biomédico, com ênfase na doença e não na saúde, representa apenas parte das formas possíveis de intervenção social na saúde pública, que incluem também a abordagem educativa, de mudança de comportamento, a abordagem centralizada no cliente e a abordagem societária. Programas de educação em saúde visam desencadear mudanças de comportamento individual e servir de base para a instalação de promoção de saúde, cujo intuito principal é o de promover mudanças no comportamento organizacional $^{(24)}$.

O esforço preventivo é amplamente justificado uma vez que a orientação correta configura-se em medida de prevenção de cegueira e de transtornos visuais. Ressalta-se que a cegueira e a incapacidade visual acarretam conseqüências sociais, psicológicas e econômicas adversas para o indivíduo e para a sociedade ${ }^{(25)}$.

\section{CONCLUSÃO}

Revelou-se insuficiência de conhecimentos em relação ao glaucoma, às formas de prevenção e de tratamento. Esse fato sugere a necessidade de ações educativas visando à divulgação de conhecimentos relativos ao glaucoma, entre os pacientes e a população em geral, como forma de prevenção da perda visual.

\section{ABSTRACT}

Purpose: To verify information related to glaucoma and level of self-evaluation of knowledge about the disease, in order to help doctor-patient relationship and to stimulate the observance regarding treatment. Methods: In the Provincial Public Hospital, São Paulo, Brazil, a cross-sectional survey was performed by applying a questionnaire. The variable "self-evaluation of the knowledge" was measured by an ordinal scale (to know well, to know more or less, to know badly and knowing nothing). Results: The population was consisted of 405 patients with glaucoma; $72.6 \%$ female; mean age 66.17 years. Of those who "know well" about the control of the disease, $95.8 \%$ declared having received explanations $(\mathrm{p}<0.000)$; there was a larger proportion of patients who stated "know more or less" when compared to other groups ( $p<0.000)$; eye specialist as the source of information on glaucoma was mentioned by $50 \%$ of the patients. Conclusion: The patients' knowledge in regarding glaucoma was related to the level of received explanations. The level of knowledge about the disease was directly related to the educational level. This study confirms the need of maintenance of guide lines, continuous popularization of information on prevention, which would help to treat patients with this problem and improve visual prognosis.

Keywords: Glaucoma/therapy; Glaucoma/prevention \& control; Patient education; Public health/education; Blindness/ prevention \& control; Hospitals, public

\section{REFERÊNCIAS}

1. Gerali PS, DiVerde M. Glaucoma high risk alert. J Ophthalmic Nurs Technol. $1991 ; 10(1): 34$.

2. Cintra FA, Costa VP, Tonussi JAG, Jose NK. Avaliação de programa educativo para portadores de glaucoma. Rev Saúde Pública. 1998; 32(2):172-7.

3. Van Buskirk EM, Cioffi GA. Glaucomatous optic neuropathy. Am J Ophthalmol. 1992; 113(4):447-52. Review.

4. Drance SM. Bowman Lecture. Glaucoma-changing concepts. Eye. 1992; 6(Pt4): 337-45.

5. Gullo RM, Costa VP, Bernardi L, Kara-José N. Condições visuais de pacientes glaucomatosos em um hospital universitário. Arq Bras Oftalmol. 1996; 59(2): $147-50$.

6. Ashburn FS Jr, Goldberg I, Kass MA. Compliance with ocular therapy. Surv Ophthalmol. 1980; 24(4):237-48. Review. 
7. Weinreb RN. Compliance with medical treatment of glaucoma. J Glaucoma. 1992, 1:134-6.

8. Costa VP, Vasconcelos JPC, Pelegrino M, José NK. O que os pacientes sabem sobre glaucoma? Arq Bras Oftalmol. 1995; 58(1):36-41.

9. Green LW, Kreuter MW. Health promotion planning - an educational and environmental approach. 2nd ed. Mountain View; CA: Mayfield; 1991. p. 54-173.

10. Temporini ER, Kara-José N. Níveis de prevenção de problemas oftalmológicos: propostas de investigação. Arq Bras Oftalmol. 1995; 58(3):189-92.

11. Temporini ER, Kara-José N, Gondim EL, Dantas FJ. Conhecimentos sobre saúde ocular entre profissionais de um hospital universitário. Medicina (Ribeirão Preto). 2002; 35(1):53-61.

12. Temporini ER. Ação preventiva em problemas visuais de escolares. Rev Saúde Pública. 1984; 18(3):259-62.

13. Piovesan A, Temporini ER. Pesquisa exploratória: procedimento metodológico para o estudo de fatores humanos no campo da saúde pública. Rev Saúde Pública. 1995; 29(4):318-25.

14. Quigley HA. Number of people with glaucoma worldwide. Br J Ophthalmol. 1996; 80(5):389-93. Review.

15. Sommer A, Tielsch JM, Katz J, Quigley HA, Gottsch JD, Javitt JC, et al Racial differences in the cause-specific prevalence of blindness in east Baltimore. N Engl J Med. 1991; 325(20):1412-7.
16. Jose NK, Contreras F, Campos MA, Delgado AM, Mowery RL, Ellwein LB. Screening and surgical results from cataract free-zone projects in Campinas, Brazil and Chimbote, Peru. Int Ophthalmol. 1990; 14(3):155-64.

17. Chiang YP, Bassi LJ, Javitt JC. Federal budgetary costs of blindness. Milbank Q. 1992; 70(2):319-40.

18. Javitt JC, Aiello LP, Bassi LJ, Chiang YP, Canner JK. Detecting and treating retinopathy in patients with type I diabetes mellitus. Saving associated with improved implementation of current guidelines. Ophthalmology. 1991; 98(10):1565-74.

19. Zimmerman TJ, Zalta AH. Facilitating patient compliance in glaucoma therapy. Surv Ophthalmol. 1983; 28 (Suppl):252-8.

20. Ley P. Psychology of compliance. Br J Soc Clin Psychol. 1979, 18:254-8.

21. Rosenthal AR, Zimmerman JF, Tanner J. Educating the glaucoma patient. Br J Ophthalmol. 1979; 67(12):814-7.

22. Kim S et al. Glaucoma patient education. Invest Ophthal Vis Sci. 1996; 37(Suppl):S642.

23. Temporini ER. Pesquisa de oftalmologia em saúde pública: considerações metodológicas sobre fatores humanos. Arq Bras Oftalmol. 1991; 54(6):279-81.

24. Candeias NMF. Conceitos de educação e de promoção em saúde: mudanças individuais e mudanças organizacionais. Rev Saúde Pública. 1977;31(2):209-13.

25. World Health Organization. Strategies for the prevention of blindness in national programmes - a primary health care approach. Geneva: WHO; 1984

\section{Congresso Internacional de Offalmologia XXVI Congresso Pan-Americano de Oftalmologia XVII Congresso Brasileiro de Prevenção da Cegueira e Reabilitação Visual}

\section{9 a 24 de fevereiro de 2006 Transciméricc Expo-Center SÃO PAULO - SP}

INFORMAÇÕES: Meeting Eventos

Fone: (1 1 ) 3849-8263 - Fax: (1 1 ) 3849-0379

E-mail: info@ophthalmology2006.com.br

Home-page: www.ophthalmology2006.com.br 